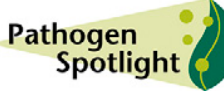

\title{
Phytopathology
}

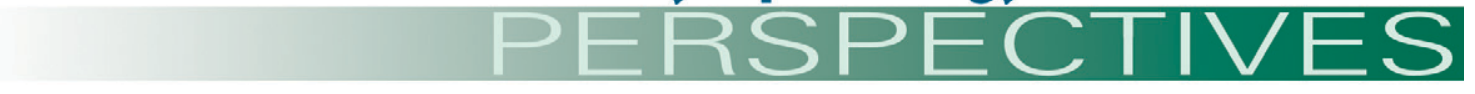

\section{One Cut to Change Them All: CRISPR/Cas, a Groundbreaking Tool for Genome Editing in Botrytis cinerea and Other Fungal Plant Pathogens}

\author{
Matthias Hahn ${ }^{1, \dagger}$ and Gabriel Scalliet ${ }^{2}$ \\ ${ }^{1}$ University of Kaiserslautern, Department of Biology, Kaiserslautern, Germany \\ ${ }^{2}$ Syngenta Crop Protection AG, Stein, Switzerland \\ Accepted for publication 29 September 2020.

\begin{abstract}
CRISPR/Cas is a genome editing technology that has opened new dimensions in functional biology. In a recent publication, we presented a highly efficient CRISPR/Cas technique for Botrytis cinerea, which dramatically increases our options to mutagenize and modify single or multiple genes. In this Perspectives article, we describe the essential features of the method and demonstrate with several examples how it opens new avenues for unraveling the virulence mechanisms of Botrytis and other plant pathogenic fungi and can accelerate research for the identification of new antifungal compounds.
\end{abstract}

Keywords: disease control and pest management, fungal pathogens, genetics, genomics, molecular, mutagenesis

The generation of mutants is a fundamental tool for functional genetics and mechanistic analysis of fungal virulence. Targeted mutagenesis by use of selection markers placed between two flanking regions for directed homology-dependent recombination (HR) has been established for filamentous fungi since the late 1980s (Fincham 1989). The key of CRISPR/Cas-mediated editing is the targeted introduction of a double-stranded DNA break by the Cas9 endonuclease loaded with a single guide RNA (gRNA), forming a ribonucleoprotein (RNP) complex. DNA breaks create an immediate, life-threatening damage that must be instantly repaired by the cell in order to survive. Two major chromosomal repair pathways exist, nonhomologous end joining (NHEJ) and HR, and their activation at targeted loci dramatically increases the frequency and thus the efficiency by which targeted changes can be introduced in the genome (Fig. 1) (Wright et al. 2016).

The standard transformation protocol for Botrytis cinerea, originally developed by Hamada et al. (1994), involves the generation of protoplasts and the addition of DNA in an osmotically stabilized medium containing polyethylene glycol. This protocol, still being used with some modifications in many fungi, is fairly efficient and allows the generation of knockout mutants when performed with resistance cassettes (e.g., hygromycin, fenhexamid,

${ }^{\dagger}$ Corresponding author: M. Hahn; hahn@biologie.uni-kl.de

The author(s) declare no conflict of interest.

(C) 2021 The American Phytopathological Society or nourseothricin) flanked with homology flanks of 0.5 to $1 \mathrm{~kb}$ for targeting the gene of interest. An advantage of $B$. cinerea is its high rate of HR, which usually allows the identification of correct transformants among the background of ectopic mutants, with rates $>10 \%$. However, a major drawback of Botrytis and related genera is their multinuclear nature, which imposes tedious rounds of cultivation for single-spore isolation after transformation to achieve homokaryosis.

CRISPR/Cas-based transformation methods have been described for a large number of filamentous fungi, including multiple plant pathogens (Schuster and Kahmann 2019). In most cases, they are based on the transgenic expression of both Cas9 and the gRNA. As an alternative, which is less commonly used, Cas9 and the gRNA can be assembled to a functional RNP in vitro and subsequently added as purified RNP in the transformation, as was demonstrated for Magnaporthe oryzae (Foster et al. 2018). We have used this strategy to develop a powerful CRISPR/Cas method for $B$. cinerea (Leisen et al. 2020). Technically, it follows the standard transformation protocol, but instead of a knockout cassette, purified Cas9 protein and gRNAs are added with one or more DNAs as repair templates to the protoplasts. Because of its limited half-life within cells, the use of RNP simplifies the transformation protocol and reduces the risk of off-target mutations. This finding was confirmed by genome sequencing, which revealed less than five undesired mutations in the edited strains (Leisen et al. 2020). Our CRISPR/ Cas protocol increased marker-assisted, targeted mutagenesis to about $90 \%$ efficiency (most transformants resulted from integration via HR), and homology flanks can be reduced to $60 \mathrm{bp}$, allowing the rapid single-step PCR-based synthesis of knockout constructs. This 
mutagenesis approach is flexible: By modifying the homology flanks, a targeted insertion and a 2-kb deletion could be generated with similar efficiency. This approach opens the possibility of deleting larger genomic regions or multiple genes in a single step. In Fusarium proliferatum, deletion of an 8-kb genomic fragment encoding a polyketide synthase involved in mycotoxin biosynthesis has been recently achieved, using Cas9-RNPs with two gRNAs (Ferrara et al. 2019). The high targeting efficiency allowed the generation of double mutants in a single transformation assay, by using two gRNAs and repair templates with different selection markers (Leisen et al. 2020).

The need for a resistance cassette in the transforming DNA severely restricts the options for multiple genetic manipulations and excludes the introduction of small changes, such as point mutations, without altering the structure of the targeted locus. To further develop CRISPR/Cas into an editing tool with a maximum degree of flexibility and freedom of design, we took advantage of cotransformation and coediting. It has been known for a long time that simultaneous transformation of two or more DNAs occurs with much higher frequency than expected from independent transformation events (Wernars et al. 1987). Therefore, if two different transformation events are combined (e.g., two CRISPR editings or plasmid transformation coupled with CRISPR editing), selection has to be applied for only one event, and the transformants are subsequently screened for the other. Two strategies exploiting this principle were designed for markerfree editing. The most efficient strategy for $B$. cinerea involves cotransformation of the CRISPR/Cas components with an autonomously replicating telomere vector (pTEL). Transformants carrying the resistance marker of the vector are screened for the desired editing events, and the unstable pTEL is subsequently removed from the edited transformants simply by stop of selection. As a result, nothing but the desired editing event has occurred in the transformants. This approach worked efficiently in a variety of applications (Fig. 2) (Leisen et al. 2020). Using cotransformation with pTEL, we were able to generate markerfree knockouts (either by NHEJ or HR) and to generate a knock-in fusion of superoxide dismutase 1 (Sod1) and GFP with astonishing efficiency. Among thousands of transformants, $>60 \%$ showed fluorescence and were confirmed to have correctly integrated the fusion construct. Fluorescent labeling of proteins of interest can

\section{FIGURE 1}

Comparison of traditional and CRISPR/Cas-directed knockout strategies in filamentous fungi. Traditional strategies require relatively large homology flanks to promote homologous recombination (HR), but ectopic integrations still predominate. With CRISPR/Cas, the double-stranded cut of the target DNA boosts HR-mediated integration, even when short homology flanks are used.

\footnotetext{
FIGURE 2

Schematic illustrations of marker-free editings via cotransformation of pTEL that have been successfully performed in $B$. cinerea (Leisen et al. 2020). The scissors indicate the sites of Cas9-guide RNAribonucleoprotein-induced DNA breaks, and the crossed lines indicate the regions where homologous recombination (HR) occurs. The colored

lines represent mutations induced by nonhomologous end joining (NHEJ), or via HR of a mixed repair template in the case of multiediting. The white horizontal bar indicates a stuffer fragment inserted between the short homology flanks to facilitate PCR-based detection of deletion mutants.
}

now be used as a routine tool to study their expression and subcellular localization, by changing the endogenous gene locus. Given the effectiveness of these knock-in events, we foresee an expansion of cell biology studies that will use protein tagging such as the in vivo monitoring of protein-protein interaction at the subcellular level via bimolecular fluorescence complementation or similar methods (Okabe et al. 2018). Another powerful use of marker-free mutagenesis is reverse chemical genetics, such as the modification of protein kinases to render them sensitive to adenosine triphosphate analogs (Elphick et al. 2009). These minor active site modifications do not affect essential signaling function of the kinases and enable conditional functional knockout to be performed in planta by chemical treatment. For example, introduction of an adenosine triphosphate analog-sensitive version of the mitogen-activated protein kinase PMK1 allowed the demonstration of its role in cell-to-cell mobility of Magnaporthe oryzae (Sakulkoo et al. 2018). With CRISPR/Cas, it will be easy to generate a set of conditionally inactive mitogen-activated protein kinases to address their multiple functions in greater depth. Marker-free mutations can also be introduced via cotransformation with high rates by means of error-prone NHEJ repair, without repair template and HR. Although being less precise, it works efficiently for gene knockouts by inducing frameshift mutations, as shown for Bos1 (Leisen et al. 2020). For introduction of NHEJ-based deletions, mixtures of two RNPs targeting two loci in the same gene have been used efficiently for gene knockouts in Magnaporthe oryzae (A. Foster, personal communication).

With CRISPR/Cas, multiple independent transformants with the correct genotype are easily obtained. This method greatly increases the speed and accuracy of mutant analysis. Complementation of a knockout mutant is needed as final proof for gene function. This step is normally done by ectopic introduction of a wild-type (WT) gene copy into the mutant, but incomplete restoration of the mutant phenotype in the revertant can occur because expression levels of the gene in focus may differ from the original WT copy. Functional validations can be improved with CRISPR/Cas via pTEL, which allows a markerless replacement of the mutant allele by a functional copy at the same genomic locus. In this case, a functionally neutral sequence tag should be integrated into the complementing allele to distinguish the revertant from contaminating WT cells.
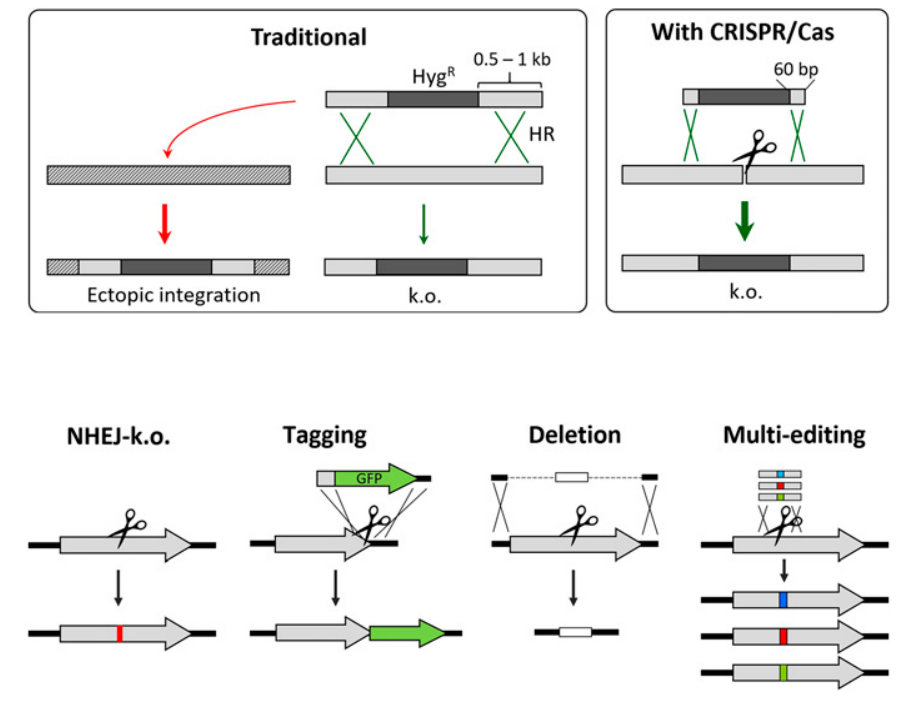
B. cinerea is a pathogen with an exceptionally large host range (Elad et al. 2016). It is not yet fully understood what makes this fungus such a ubiquitous and indiscriminate killer. During host invasion, it secretes a cocktail of cell wall degrading enzymes, phytotoxic proteins, and metabolites. Successful infection is accompanied by induction of programmed host cell death, associated with strong defense responses, which seem ineffective against necrotrophic pathogens (Govrin and Levine 2000; Veloso and van Kan 2018). Often, phenotypes of mutants provide no clear evidence about their role in the infection process. For only a few cell wall degrading enzymes, such as the polygalacturonases PG1 and PG2, a function in virulence has been confirmed (Kars et al. 2005). For two xylanases (Xyn11A and Xyl1), their involvement in pathogenesis is based on HR-inducing structural patterns that are not linked to their enzymatic activity (Noda et al. 2010; Yang et al. 2018). The apparent redundancy of virulence factors demands a comprehensive analysis of protein families with similar functions, such as polygalacturonases and aspartic proteases (Kars et al. 2005; ten Have et al. 2010). An effective multiple knockout approach is available now, by applying pTEL- or marker exchange-assisted editing in a repetitive manner. We are currently testing the efficiency of pTEL cotransformation to introduce two or three marker-free mutations in one step. After genetic confirmation of the editing events and purification of homokaryons, the transformants can be used directly for the next round of mutagenesis. Multiple targeting would also allow us to address questions related to functional aspects of repeated DNA, such as transposable elements. In a seminal work, Weiberg et al. (2013) demonstrated that $B$. cinerea releases small interfering RNAs during infection, which contribute to pathogenesis by inhibiting host defense genes. Noteworthy, many of these small interfering RNAs are derived from replicase genes of active retroelements, which are present in multiple copies, such as the LTR retrotransposon Boty (Diolez et al. 1995). Because CRISPR/Cas enables the simultaneous targeting of multiple repetitive loci, functional investigations of these elements should be possible now.

Another important application is the modification of proteins for structure and function investigations, as exemplified for fungicide target proteins. We could show how pTEL cotransformation enabled the one-step exchange of a single resistanceconferring codon for its 20 potential amino acids (Leisen et al. 2020). The mutant library was generated via a cotransformation approach combining ribonucleoprotein, bulked synthetic repair templates encoding each of the 20 amino acids, and pTEL for selection. Using amplicon sequencing, we monitored the presence of the mutant types and their relative abundance in the mutant library before and after fungicide selection. Although all 20 mutations were detected among the edited transformants in the absence of selection, several amino acids were positively selected by fungicides of the succinate dehydrogenase inhibitor family, whose prevalence agreed with the cross-resistance profiles displayed by the individual mutants (Leisen et al. 2020). This genome editing approach enables functional screens using bulked populations of genetically engineered variants. This method is novel for plant pathogens and could find a broad range of applications in all situations where positive selection can be applied. These encompass variants screens for evaluating fungicide resistance risk or supporting drug design and search for mutations or protein variants leading to increased performance in vitro or during infection. For these applications, it might be useful to mark the mutants with unique bar codes, which would simplify their identification and allow us to study their performance through competition experiments (Eason et al. 2004). Increased editing efficiency even enables multilocus variant approaches, which are particularly relevant for the experimental assessment of combinations of variants affecting multiple genes. These assessments could complement population genomics investigations such as genome-wide association studies to reveal fitness interdependencies, which are relevant for resistance evolution. The identification of an Achilles heel defeating resistance evolution could have practical implications in the control of Botrytis cinerea, which is the plant pathogen that has developed by far the most impressive multi-fungicide-resistant genotypes (Rupp et al. 2016).

CRISPR/Cas technologies are being developed further at a rapid pace. A wide range of class 2 Cas endonucleases are available now, including engineered variants or variants originating from different organisms. Modified Cas9 versions displaying altered protospacer adjacent motif (PAM) recognition sites (e.g., NGA or NAG instead of NGG; Kleinstiver et al. 2015), and alternative endonucleases such as Cpf1 recognizing a TTTN PAM sequence (Vanegas et al. 2019) enable an expanding targeting scope, facilitating the editing of a larger number of genomic loci. The use of Cpf1 seems particularly relevant for Botrytis cinerea, which carries an AT-rich genome. For applications involving explorative mutagenesis of a gene, alternatives to the synthetic repair template library previously described have been developed, such as base editors, which are nuclease-deficient Cas9 derivates fused to cytidine or deoxyadenosine deaminases. Classic base editors are able to mutagenize a small window of positions close to the PAM site without double-stranded break formation or the need for repair templates (Anzalone et al. 2020). Of particular note is CRISPR-X, which targets variants of cytidine deaminase by using MS2-modified gRNAs and enables the mutagenesis of a 100-bp window surrounding the gRNA target site (Hess et al. 2016). More recently, prime editing (PE) has been developed, which may deliver vastly improved throughput for fully rationalized mutagenesis (Anzalone et al. 2019). PE is based on the fusion of the Cas9 nickase domain with an engineered reverse transcription domain. The PE guide RNA contains an extension encoding the desired edit, so that all possible point mutations can be introduced through rational synthesis of gRNA variants without the need for a repair template or HR. Provided the respective fusion proteins can be produced and transfected in filamentous fungi, both base and prime editing strategies offer great promise for RNP-based targeted mutagenesis.

A problem that remains to be overcome for a truly highperformance mutagenesis platform in Botrytis cinerea is the time needed for purification of homokaryotic transformants. Ideally, this would be achieved by positive selection of mutated nuclei and negative selection of WT nuclei, leading to their removal. Before such a selection is available, the use of marker switching is a robust strategy, because it allows the selection of edited transformants and checking for the loss of the marker in the "WT" recipient. We think that the effectiveness of our editing strategy could support miniaturization and permit the generation of a large $B$. cinerea mutant collection available to the scientific community. Systematic genome-wide knockout sets have been so far obtained for Neurospora crassa (Park et al. 2011) and Aspergillus fumigatus (https://www.phe-culturecollections.org.uk/news/ncpf-news/the-aspergillus-fumigatus-genome-wideknockout-collection.aspx) two filamentous fungi that are not pathogens of plants. Considering the performance of our CRISPR approach, we believe that generating such a highly valuable collection may become manageable by a coordinated effort. This seems a worthwhile strategic goal to achieve for the gray mold pathogen, given the major gaps in addressing gene function in this important pathogen and the need to safeguard food production for our growing population.

\section{ACKNOWLEDGMENTS}

We thank Felix Willmund, David Scheuring, and Thomas Leisen for their comments on the manuscript, and Andrew Foster for inspiring discussions. 


\section{LITERATURE CITED}

Anzalone, A. V., Koblan, L. W., and Liu, D. R. 2020. Genome editing with CRISPR-Cas nucleases, base editors, transposases and prime editors. Nat. Biotechnol. 38:824-844.

Anzalone, A. V., Randolph, P. B., Davis, J. R., Sousa, A. A., Koblan, L. W., Levy, J. M., Chen, P. J., Wilson, C., Newby, G. A., Raguram, A., and Liu, D. R. 2019. Search-and-replace genome editing without double-strand breaks or donor DNA. Nature 576:149-157.

Diolez, A., Marches, F., Fortini, D., and Brygoo, Y. 1995. Boty, a longterminal-repeat retroelement in the phytopathogenic fungus Botrytis cinerea. Appl. Environ. Microbiol. 61:103-108.

Eason, R. G., Pourmand, N., Tongprasit, W., Herman, Z. S., Anthony, K., Jejelowo, O., Davis, R. W., and Stolc, V. 2004. Characterization of synthetic DNA bar codes in Saccharomyces cerevisiae gene-deletion strains. Proc. Natl. Acad. Sci. USA 101:11046-11051.

Elad, Y., Pertot, I., Prado, A. M. C., and Stewart, A. 2016. Plant hosts of Botrytis spp. In: Botrytis: The Fungus, the Pathogen and Its Management in Agricultural Systems. S. Fillinger and Y. Elad, eds. Cham, Switzerland

Elphick, L. M., Lee, S. E., Anderson, A. A., Child, E. S., Bonnac, L., Gouverneur, V., and Mann, D. J. 2009. Exploring the roles of protein kinases using chemical genetics. Future Med. Chem. 1:233-241.

Ferrara, M., Haidukowski, M., Logrieco, A. F., Leslie, J. F., and Mulè, G. 2019. A CRISPR-Cas9 system for genome editing of Fusarium proliferatum. Sci. Rep. 9:19836.

Fincham, J. R. 1989. Transformation in fungi. Microbiol. Rev. 53:148-170.

Foster, A. J., Martin-Urdiroz, M., Yan, X., Wright, H. S., Soanes, D. M., and Talbot, N. J. 2018. CRISPR-Cas9 ribonucleoprotein-mediated co-editing and counterselection in the rice blast fungus. Sci. Rep. 8:14355.

Govrin, E. M., and Levine, A. 2000. The hypersensitive response facilitates plant infection by the necrotrophic pathogen Botrytis cinerea. Curr. Biol. 10:751-757.

Hamada, W., Reignault, P., Bompeix, G., and Boccara, M. 1994. Transformation of Botrytis cinerea with the hygromycin B resistance gene, hph. Curr. Genet. 26:251-255.

Hess, G. T., Frésard, L., Han, K., Lee, C. H., Li, A., Cimprich, K. A., Montgomery, S. B., and Bassik, M. C. 2016. Directed evolution using dCas9-targeted somatic hypermutation in mammalian cells. Nat. Methods 13:1036-1042.

Kars, I., Krooshof, G. H., Wagemakers, L., Joosten, R., Benen, J. A. E., and van Kan, J. A. L. 2005. Necrotizing activity of five Botrytis cinerea endopolygalacturonases produced in Pichia pastoris. Plant J. 43: 213-225.

Kleinstiver, B. P., Prew, M. S., Tsai, S. Q., Topkar, V. V., Nguyen, N. T., Zheng, Z., Gonzales, A. P. W., Li, Z., Peterson, R. T., Yeh, J.-R. J., Aryee, M. J., and Joung, J. K. 2015. Engineered CRISPR-Cas9 nucleases with altered PAM specificities. Nature 523:481-485.
Leisen, T., Bietz, F., Werner, J., Wegner, A., Schaffrath, U., Scheuring, D., Willmund, F., Mosbach, A., Scalliet, G., and Hahn, M. 2020. CRISPR/Cas with ribonucleoprotein complexes and transiently selected telomere vectors allows highly efficient marker-free and multiple genome editing in Botrytis cinerea. PLoS Pathog. 16:e1008326.

Noda, J., Brito, N., and González, C. 2010. The Botrytis cinerea xylanase Xyn11A contributes to virulence with its necrotizing activity, not with its catalytic activity. BMC Plant Biol. 10:38.

Okabe, T., Katayama, T., Mo, T., Mori, N., Jin, F. J., Fujii, I., Iwashita, K., Kitamoto, K., and Maruyama, J.-I. 2018. BiFC-based visualisation system reveals cell fusion morphology and heterokaryon incompatibility in the filamentous fungus Aspergillus oryzae[published correction appears in Sci. Rep. 2019;9:16078]. Sci. Rep. 8:2922.

Park, G., Colot, H. V., Collopy, P. D., Krystofova, S., Crew, C., Ringelberg, C., Litvinkova, L., Altamirano, L., Li, L., Curilla, S., Wang, W., Gorrochotegui-Escalante, N., Dunlap, J. C., and Borkovich, K. A. 2011. High-throughput production of gene replacement mutants in Neurospora crassa. Methods Mol. Biol. 722:179-189.

Rupp, S., Weber, R. W. S., Rieger, D., Detzel, P., and Hahn, M. 2016. Spread of Botrytis cinerea strains with multiple fungicide resistance in German horticulture. Front. Microbiol. 7:2075.

Sakulkoo, W., Osés-Ruiz, M., Oliveira Garcia, E., Soanes, D. M., Littlejohn, G. R., Hacker, C., Correia, A., Valent, B., and Talbot, N. J. 2018. A single fungal MAP kinase controls plant cell-to-cell invasion by the rice blast fungus. Science 359:1399-1403.

Schuster, M., and Kahmann, R. 2019. CRISPR-Cas9 genome editing approaches in filamentous fungi and oomycetes. Fungal Genet. Biol. 130: 43-53.

ten Have, A., Espino, J. J., Dekkers, E., van Sluyter, S. C., Brito, N., Kay, J., González, C., and van Kan, J. A. L. 2010. The Botrytis cinerea aspartic proteinase family. Fungal Genet. Biol. 47:53-65.

Vanegas, K. G., Jarczynska, Z. D., Strucko, T., and Mortensen, U. H. 2019. Cpf1 enables fast and efficient genome editing in Aspergilli. Fungal Biol. Biotechnol. 6:6

Veloso, J., and van Kan, J. A. L. 2018. Many shades of grey in Botrytis-host plant interactions. Trends Plant Sci. 23:613-622.

Weiberg, A., Wang, M., Lin, F.-M., Zhao, H., Zhang, Z., Kaloshian, I., Huang, H.-D., and Jin, H. 2013. Fungal small RNAs suppress plant immunity by hijacking host RNA interference pathways. Science 342:118-123.

Wernars, K., Goosen, T., Wennekes, B. M., Swart, K., van den Hondel, C. A., and van den Broek, H. W. 1987. Cotransformation of Aspergillus nidulans: A tool for replacing fungal genes. Mol. Gen. Genet. 209:71-77.

Wright, A. V., Nuñez, J. K., and Doudna, J. A. 2016. Biology and applications of CRISPR systems: harnessing nature's toolbox for genome engineering. Cell 164:29-44.

Yang, Y., Yang, X., Dong, Y., and Qiu, D. 2018. The Botrytis cinerea xylanase BcXyl1 modulates plant immunity. Front. Microbiol. 9:2535. 